\title{
Stuck in Graduate Medical Education Traffic? Teaching Health Centers Are Family Medicine's High-Occupancy-Vehicle Lane
}

\author{
Joseph W. Gravel, Jr., MD
}

In this issue of the fournal, Levin and colleagues ${ }^{1}$ report that family physicians trained in Teaching Health Centers-predominately residency programs sponsored by Community Health Centers (CHCs) were twice as likely to intend to continue working in these settings compared with those trained in other settings. This finding is remarkably consistent with an earlier study ${ }^{2}$ that showed CHC-trained family physicians were 2.7 times more likely to work in underserved settings than non-CHC-trained family physicians.

Irrespective of its insurance reform and access provisions, the Affordable Care Act in 2010 was a necessary workforce intervention for the underserved. Before the Teaching Health Center (THC) pilot, the proportion of residencies providing continuity training in CHCs had remained unchanged since 1992. ${ }^{3}$ The THC program is small but mighty-representing less than $0.5 \%$ of total governmental funding spent to train physicians, but producing (including those in training) over 1000 primary care physicians and dentists for communities that would otherwise go without. ${ }^{4}$ More than half of teaching health centers $(57 \%)$ are located in states in the lowest 4 quintiles of Medicare-funded resident-to-population ratio, and more than $70 \%$ are located in federally designated high-need areas. ${ }^{5}$

The central concept of high-occupancy-vehicle (HOV) lanes is that given a finite resource (the number of built traffic lanes) it is most important to move more people, not more cars-an outcomes rather than a process measure. The Balanced Budget Act of 1997 limiting Medicare-funded residency positions without regard to specialty type effectively froze the number of lanes on the road

Funding: none.

Conflict of interest: none declared.

Corresponding author: Joseph W. Gravel, Jr., MD, Greater Lawrence Family Health Center, 34 Haverhill Street, Lawrence, MA 01841 (E-mail: jgravel@glfhc.org). without addressing the types of vehicles being produced. Despite numerous calls for Medicare Graduate Medical Education (GME) funding reform, ${ }^{6-10}$ hospitals are still subsidized to produce whatever they want regardless of societal need. In contrast, $91 \%$ of THC graduates remain in primary care practice (only 23\% for Medicare GME), most practice in underserved communities after graduation ( $80 \%$ vs $26 \%$ in Medicare GME), and $19 \%$ practice in rural America compared with only $8 \%$ of Medicare GME graduates. ${ }^{6}$

The good news is there is an accessible way for family medicine to circumvent seemingly intractable Medicare GME funding reform gridlock. The HOV lanes (CHCs) have already been built and are educationally rich but still educationally underutilized. Signage (and sometimes a bit more) is needed to convert a highway lane into an $\mathrm{HOV}$ lane; $\mathrm{CHCs}$ not currently training family physicians similarly need some additional work to become effective THCs. A relatively small amount of dependable funding and technical assistance are the essential ingredients. Just as commuters need to know the HOV lane will be dependably open and affordable before rearranging for carpooling, continued funding uncertainty makes investment in the THC model nearly impossible for most CHCs.

For HOV lanes to work, there also must be restrictions placed on their use and penalties for improper use. Similarly, THCs must continue to be precisely defined and eligibility criteria regulated to assure that primary care is what is being supported and produced rather than becoming an ancillary backdoor for those who historically have been dominating the other lanes. HOV lanes indirectly benefit everyone (by reducing emissions); THCs reduce emergency department visits and overall health care costs that affect private insurance premiums and taxes to support governmental insurers. HOV lanes work best when there are 
large numbers of people in buses—similarly, expansion of existing THCs with a reliable funding mechanism would provide economies of scale.

\section{Training Advantages of CHCs for Family Medicine (FM)}

The THC program dovetails nicely with family medicine's own workforce goals and training needs. The American Academy of Family Physicians (AAFP's) ambitious " $25 \times 2030$ " initiative $(25 \%$ of residency positions will be in FM by 2030) will require a large increase in family medicine residency training positions. There are currently $1400 \mathrm{CHCs}$ in the United States (44\% in rural areas) ${ }^{11}$ and only 59 THCs, highlighting the potential growth opportunity. CHC-based residents treat an enhanced severity and diversity of diseases in these patient populations. They learn cost-effective practice that likely mirrors (to the better) academic health centers' "imprinting" phenomenon. ${ }^{12}$ Cost-effective pharmacologic prescribing is learned by necessity. There is often less "learned helplessness" that can pervade overly specialist-rich primary care training environments. Poverty's associated behavioral health issues provides residents a significant experience. Other skill sets include working closely with social workers, language skills (eg, to serve the rapidly growing Spanishspeaking demographic in the United States), population health, and office-based procedures, a particular necessity for patients with limited access to specialists. $\mathrm{CHCs}$ are the outpatient equivalent of an interprofessional inpatient hospital training environmentclinical pharmacists, integrated behavioral health, social services, and even dental services are colocated in many CHCs.

Given adequate resources, a $\mathrm{CHC}$ is an excellent mechanism for the reintegration of medicine and public health to address social determinants of health. CHCs are a precisely targeted socioeconomic intervention for America's most distressed communities. THCs are even more effective as they bring young professionals (and their families) to communities that have often experienced outmigration of this particular socioeconomic group and help retain them there. GME provides numerous other direct and indirect economic benefits to communities. ${ }^{13}$ Combining CHCs and GME (to create a THC) is a synergistic construct that helps repair a fraying social contract, benefiting all.

Not all CHC's can or should serve as THCs, and $\mathrm{CHCs}$ are in varying degrees of readiness to sponsor residencies. Besides AAFP's and the Council of Aca- demic FM's current advocacy efforts, ${ }^{14,15}$ family medicine's academic organizations should make collaborating with $\mathrm{CHCs}$ a higher strategic priority, respecting the differences in cultures that need to be navigated to be successful. In addition to GME, FM departments facilitating $\mathrm{CHC}$ experiences will increase the likelihood of practicing in underserved communities. ${ }^{16,17}$ The American Board of Family Medicine can play an important role in this transformation effort with its focus on quality, standard setting, professionalism, and integration of a physician's development during GME with ongoing professional development when in practice.

\section{How Do We Prepare CHCs for a Greater Educational Mission?}

Effective THCs require a shared mission of service and education. CHC CEOs and Boards of Directors often do not fully comprehend the inherent inefficiencies that educating physicians, particularly first year residents cause. Governance, complex governing institutional regulations and administrative challenges, chronic underfunding, and leadership issues are the most important barriers to effective CHC-Family Medicine Residency (FMR) partnerships or sponsorships. ${ }^{18,19}$ Compared with non-CHC physicians, CHC physicians are less satisfied with their employers, ${ }^{20}$ which may impact recruitment and retention. It is important for this to be further studied to better identify the causes of dissatisfaction. Although certainly not unique to CHCs, working in cultures with reduced professional autonomy is problematic not only for physicians and residents but ultimately the $\mathrm{CHC}$ itself.

In addition, a more formalized and well-advertised, low-cost consultation service staffed by volunteer CHC-experienced educators could be created to work with $\mathrm{CHCs}$ to assess readiness and provide ongoing technical assistance, similar to family medicine's Residency Program Solutions. Establishing an academically focused service by the American Association of Teaching Health Centers (AATHC) would be a natural next step in its evolution. More formalized advocacy collaborations (standing meetings, cross-pollination at national organization meetings, establishing liaisons, etc.) between family medicine (AAFP and Council of Academic Family Medicine), the AATHC, and the National Association of Community Health Centers (CHCs) could help grow the THC movement for mutual benefit. 


\section{Making the THC Program Permanent}

Despite proven effectiveness and bipartisan support for THCs, the next "funding cliff" for Teaching Health Centers is October 1, 2019-the end of the program unless Congress reauthorizes another round of stopgap funding or creates permanency. The Teaching Health Center program serves as a highly successful model for broader GME funding reform, and can at least successfully coexist with the 1965 hospital-based Medicare GME funding mechanism in the meantime. Given their presence in rural and urban America, in red states and blue, THCs could even serve as a noncontroversial project to help reenergize the concept of legislative bipartisanship.

Community-based Teaching Health Center Graduate Medical Education directly addresses primary care workforce shortages, targeted to patients and communities that the Medicare GME-based system has failed. Unlike complex Medicare GME funding, THC GME's reporting requirements ${ }^{21}$ are much more transparent and accountable to the taxpayer, as every dollar is directly paid to the educational program rather than partially repurposed for other hospital needs. Current stringent eligibility requirements should be maintained to minimize the temptation to game the system to obtain funding. The current per resident payment methodology should continue to transparently cover direct and indirect expenses, currently at $\$ 150,000$ per resident based on careful studies of actual costs. ${ }^{22}$ The uncertainty of funding has hurt the program ${ }^{8}$ and a permanent funding mechanism is necessary.

Much like our CHC patients, THCs' lack of residency funding security makes sustainability difficult and longer-term investment impossible. The program should be expanded to best serve the nation's primary care needs, not only those of the underserved. Teaching Health Center growth and permanency will make Family Medicine's own growth and permanency more likely. Neither are inevitable without active advocacy now.

To see this article online, please go to: http://jabfm.org/content/ 32/2/130.full.

\section{References}

1. Levin Z, Meyers P, Peterson L, Habib A, Bazemore A. Practice intentions of family physicians trained in Teaching Health Centers: the value of community-based training. J Am Board Fam Med 2019; 32:134-35.
2. Morris CG, Johnson B, Kim S, Chen F. Training family physicians in community health centers: A health workforce solution. Fam Med 2008;40:271276.

3. Morris CG, Lesko SE, Andrilla HA, Chen FM. Family medicine residency training in community health centers: A national survey. Acad Med 2010; 85:1640-1644.

4. Health Resources \& Services Administration. Teaching health center graduate medical education program. Available from: https://bhw.hrsa.gov/grants/medicine/ thcome. Accessed January 4, 2019.

5. Barclift SC, Brown EJ, Finnegan SC, Cohen ER, Klink K. Teaching health center graduate medical education locations predominantly located in federally designated underserved areas. J Grad Med Educ 2016;8:241-243.

6. Ku L, Mullan F, Serrano C, Barber Z, Shin P. Teaching health centers: A promising approach for building primary care workforce for the 21st century. Policy research brief n0.40. Washington, DC: George Washington University Milken Institute School of Public Health. 2015. Available from: https://publichealth.gwu. edu/pdf/eIR/GGRCHN_PolicyResearchBrief_40.pdf. Accessed January 4, 2019.

7. Blanchard J, Petterson S, Bazemore A, Watkins K, Mullan F. Characteristics and distribution of graduate medical education training sites: Are we missing opportunities to meet U.S. health workforce needs? Acad Med 2016;91:1416-1422.

8. Gravel JW. Uncertainty in Teaching Health Center (THC) funding: Still crazy after all these years. J Am Board Fam Med 2017;30:275-278.

9. Iglehart JK Institute of Medicine report on GME-A call for reform. N Engl J Med 2015;372: $376-381$.

10. O'Rourke K. IOM report calls for overhaul of graduate medical education. JAMA 2014;312:880-881.

11. National Association of Community Health Centers. America's Health Centers. August 2018. http://www. nachc.org/wp-content/uploads/2018/08/AmericasHealth Centers_FINAL.pdf. Accessed February 7, 2019.

12. Chen C, Petterson S, Phillips R, Bazemore A, Mullan F. Spending patterns in region of residency training and subsequent expenditures for care provided by practicing physicians for Medicare beneficiaries. JAMA 2014;312:2385-2393.

13. Pugno PA, Gillanders WR, Kozakowski SM. The direct, indirect, and intangible benefits of graduate medical education programs to their sponsoring institutions and communities. J Grad Med Educ 2010; 2:154-159.

14. Martin S. American Academy of Family Physicians "In The Trenches" Blog. Teaching health centers funding a top priority for 2019. December 4, 2018. Available from: https://www.aafp.org/news/blogs/ inthetrenches/entry/20181204itt-thcgme.html. Accessed January 4, 2019. 
15. Hall MN, Wittenberg H. Step up and advocate for family medicine. Ann Fam Med 2012;10:177-178.

16. Guilbault RW, Vinson JA. Clinical medical education in rural and underserved areas and eventual practice outcomes: A systematic review and metaanalysis. Educ Health 2017;30:146-155.

17. Rabinowitz HK. Recruitment, retention, and follow-up of graduates of a program to increase the number of family physicians in rural and underserved areas. N Engl J Med 1993;328:934-939.

18. Sunshine JE, Morris CG, Keen M, Andrilla CH, Chen FM. Barriers to training family medicine residents in community health centers. Fam Med 2010; 42:248-254.
19. Morris CG, Chen FM. Training residents in community health centers: Facilitators and barriers. Ann Fam Med 2009;7:488-494.

20. Cole AM, Doescher M, Phillips WR, Ford P, Stevens NG. Satisfaction of family physicians working in community health centers. J Am Board Fam Med 2012;25:470-476.

21. Durfey SNM, George P, Adashi EY. Permanent GME funding for teaching health centers. JAMA 2017;317:2277-2278.

22. Regenstein M, Nocella K, Jewers MM, Mullan F. The cost of residency training in teaching health centers. N Engl J Med 2016;375:612-614. 